\title{
Thomas Blanke Antidemokratische Effekte der verfassungsgerichtlichen Demokratietheorie
}

\begin{abstract}
Von der breiteren Öffentlichkeit weitgehend unbemerkt, findet seit Jahrzebnten ein zähes Ringen um das Demokratieprinzip des GG statt. Die Auseinandersetzung geht darum, ob und wenn ja, wieviel, "Binnendemokratie im Staat, seinen Organen, den Gebietskörperschaften und Selbstverwaltungseinrichtungen sowie den öffentlichen Unternehmungen zulassig ist. Sie spaltet die politischen Lager und beschäftigt wie keine zweite die Verfassungs- und Verwaltungsgerichte in Bund und Ländern, bält die rechtswissenschaftliche Literatur in Atem und die Gesetzesproduktion am Laufen. Die Spaltung verläuft entlang einer Grenzlinie, die ansonsten zunehmend unschärfer wird: entlang der Parteigrenzen von SPD und CDU.
\end{abstract}

Die Red.

\section{Die Dogmatisierung des »Böckenförde-Konzepts" der Demokratie in der} verfassungsgerichtlichen Rechtsprechung

Das von Böckenförde ${ }^{1}$ und Herzog ${ }^{2}$ zu Beginn der 7oer Jahre entwickelte und seitdem im staatsrechtstheoretischen Diskurs äußerst einflußreiche Demokratiemodell hat sich seit Mitte der 8oer Jahre in der obergerichtlichen Rechtsprechung zunächst einiger Landesverfassungsgerichte ${ }^{3}$ sowie des Zweiten Senats des Bundesverfassungsgerichts ${ }^{*}$ und jüngst in zwei Vorlagebeschlüssen des Bundesverwaltungsgerichts v. 17. 12. $1997^{\prime}$ auf breiter Linie durchgesetzt. Diese Lesart des Demokratiekonzepts nach dem GG nimmt in ihrer dogmatisierten Gestalt zunehmend fundamentalistische Züge an. Die Beschlüsse des Zweiten Senats des BVerfG zur Verfassungswidrigkeit der Geserze der Bundesländex Hamburg ${ }^{6}$ und SchleswigHolstein, ${ }^{7}$ in denen Teilen der ausländischen Wohnbevölkerung Wahlrechte zu kommunalen Selbstverwaltungsorganen eingeräumt worden waren, fanden wegen ihres volksbezogenen Demokratiebegriffs zwar noch einige publizistische Aufmerksamkeit. $^{8}$ Demgegenüber blieb die Kette von Entscheidungen, mit denen in ebenso

\footnotetext{
1 Demokratie als Verfassungsprinzip, in: Isensee/Kirchhoff, Handbuch des Staatsrechts, Bd.I, 1987, $\$ 22$ Rn. 14, S. 887 ff.

2 Allgemeine Scaatslchre, S. 204 ff.; ders., in: Maunz/Dürig/Herzog/Scholz, GG, Art. 20 Rn. $46 \mathrm{ff}$.

3 VerfGH NW v. Is.9. 198 5, DVB! 1986, I 196ff.; HcssStGH v. 30.4. 1986, PersR 1986, 148 ff.; VerfGH RP

v. 18.4. 1994, PersR I 994,272 ff.

4 BVerfG v. 24. 5. 1995 , BVerfGE 93,37ff.

s $A z: 6 C_{1}$ und 2.97 .

6 BVerfGE 83, 60 ff.

7 BVerfGE. $83,37 \mathrm{ff}$

$8 \mathrm{Vg}$, nur Frank, Ausländerwahlrecht und Rechtsstellung der Kommunc, KJ 1990, 290 ff.
} 
beständiger wie verblüffender Regelmäßigkeit gesetzliche Regelungen zur Arbeitnehmermitbestimmung in öffentlichen Einrichtungen, die von sozialdemokratischen Parlamentsmehrheiten in den Bundesländern getroffen wurden, zumeist auf Antrag der CDU-Opposition vom Bundes- oder den Landesverfassungsgerichten' aufgehoben worden sind, ohne größere öffentliche Resonanz. Der rechtliche Vorwurf ist, von nur im Detail beachtlichen Varianten abgesehen, ${ }^{10}$ stets der gleiche: Der sozialdemokratische Gesetzgeber verkennt, zumindest was die Binnenstruktur des Staates angeht, das Demokratieprinzip. Denn die Arbeitnehmervertreter seien nicht hinreichend demokratisch legitimiert, um bei Entscheidungen, die für die Erfüllung des Amtsauftrages der Verwaltung von Bedeutung sind, mitbestimmen zu können. Insbesondere fehle ihnen für eine derartige Mitwirkung an der Ausübung von Staatsgewalt die personell-demokratische Legitimation. Diese komme nur solchen »Amtswaltern « zu, die ihre Ernennung entweder direkt durch das Volk oder das Parlament erhielten oder im Wege einer mununterbrochenen Legitimationskette a auf einen seinerseits personell legitimierten, unter Verantwortung gegenüber dem Parlament handelnden Amtsträger zurückführen könnten. Da die Arbeitnehmervertreter in ihr Amt von den Beschäftigten der Dienststelle gewählt werden, diese Bediensteten aber weder Volk noch gebietskörperschaftliches Teilvolk i.S. von Art. 20 Abs. 2, 28 Abs. x S. I GG seien, fehle es ihnen an der erforderlichen demokratischen Legitimation. Über diese, aus dem Demokratiegebot des GG herzuleitenden Konsequenzen dürfe sich auch der parlamentarische Gesetzgeber nicht hinwegsetzen.

\section{Die Vorlagebeschlüsse des BVerwG: Demokratieprinzip und Verwaltungsorganisation}

Die Vorlagebeschlüsse des Bundesverwaltungsgerichts vom 27. 1 2. 1997, "mit denen der 6. Senat die Verfahren in Klagen namhafter nordrhein-westfälischer Großunternehmen gegen Arbeitnehmermitbestimmung in den Leitungs- und Aufsichtsorganen zweier regionaler Wasserverbände ${ }^{12}$ gem. Art. 1oo Abs. I GG ausgesetzt und die Frage der Verfassungsmäßigkeit beider Wasserverbandsgesetze dem BVerfG zur Entscheidung vorgelegt hat, radikalisieren die Konsequenzen dieses Demokratiekonzepts nochmals crheblich. Denn nach Auffassung des BVerwG ist nicht lediglich die in beiden Errichtungsgesetzen, dem Emschergenossenschaftsgesetz und dem Lippeverbandsgesetz, ${ }^{13}$ vorgesehene Arbeitnehmermitbestimmung verfassungswid-

9 Vgl. oben Fn. 2 und 3 .

ro Hierzu eingehend Blanke, Das dircktive Mitbestimmungsreche der Bescháftigten in wirtschaftlichen Einrichtungen der öffentlichen Hand, PersR 1997, S. $329 \mathrm{ff} ., 332 \mathrm{ff}$.

1t Az: $6 \mathrm{C}$ i und 2.97.

12 Bereits die Entsrehungsgeschichte der beiden Wasserverbandsgesetze ist gespickt mit rechtswisscnschaftlichen Gutachten. Vgl. Stober, Wasserverbandsrecht und Arbeitnehmermitbestimmung, 1989 sowie ders., Erganzendes Rechtsgutachten z.ur Frage der verfassungsrechtlichen Zulässigkcit der Arbeitnehmermitbestimmung nach dem Emschergenossenschaftsgesetz, August 1990 (unveröff. Ms.), S. Iff;; Salzwedel, Wasserverbandsrecht und Arbeitnehmermitbestimmung - Grenzen der Gistaltungsfreiheit des Geseczgebers, in: Festschrift für Helmut Ridder, 1989 , S. $301 \mathrm{ff} . ;$ Nagel/Bauers, Mitbescimmung in öffentlichrechtlichen Unternehmen und Verfassungsrecht, 1990. Als Gutachter beteiligt waren ferner, soweit bekannt, zumindest Friauf, Puttner, Redeker, Kloepfer und Dammann; zu den seinerzcit vertretenen juristischen Auffassungen zur Zulässigkeit der Arbeitnehmermitbestimmung in Burcich der funkrionalen Selbstverwaltung sowie zur Prozeßgeschicbre und speziell der Berufungsentscheidung des OVG NRW v. 9.6. 1995, die sich eingehend und kricisch mit dem dominierenden Demokratieverständnis auseinandersetzt, vgl. Blanke, Arbeitnehmermitbestimmung in der nichtkommunalen Selbstverwaltung, PersR 1996, $222 \mathrm{ff}$.

13 EmschergenossenschaftsG v. 7. 2.1990, GV NW S. 144, LippevcrbandsG v. 7. 2. 1990, GV NW S. 162. 
rig. Vielmehr gilt dieses Verdikt für die Organisationsstruktur der Verbände insgesamt, weil den privaten Z,wangsmitgliedern, insbesondere den Grundstückseigentümern und Unternehmen, ein zu weitgehender Einfluß auf die Willensbildung ihrer Organe eingeräumt sei. Damit bestünden die Organe dieser Verbände, die Staatsgewalt ausübten, "mehrheitlich oder insgesamt aus Amtswaltern..., die nicht in ununterbrochener Legitimationskette auf das (Staats-)Volk im Sinne der Art. 20 Abs. 2, 28 Abs. I GG zurückgeführt werden können" (Umdr. S. I I). Dies sei aber wegen der von ihnen ausgeübten, überragend wichtigen und letztlich auch nicht privatisierbaren Staatsaufgaben im Bereich des Erhalts und der Pflege des Gemeinschaftsgutes Wasser ein zwingendes Verfassungsgebot.

Mit dieser "Steilvorlage" geht es nicht länger um Zulässigkeit und Grenzen der Arbeitnehmermitbestimmung in der öffentlichen Verwaltung, sondern grundsätzlich und allgcmein um die aus dem Demokratiegebot des GG zwingend abzuleitenden Organisationsstrukturen der Verwaltung. Wenn die Verfassung bei überragend wichtigen Gemeinwohlaufgaben eine staatliche Schutzpflicht dergestalt normiert, daß die Ausübung von Staatsgewalt nur durch personell-demokratisch legitimierte Amtswalter erfolgen darf, dann muß der Gesamtbereich der unmittclbaren und mittelbaren Staatsverwaltung unter Einschluß der funktionalen Selbstverwaltung unter zwei Aspekten durchgeforstet werden: Erstens ist zu klären, welchen Verfassungsrang die jeweils zu erledigenden öffentlichen Aufgaben haben. Geprüft werden muß sodann zweitens, ob die Organisationsstrukturen jeder einzelnen dieser in ihrer Fülle und in ihrem Variantenreichtum kaum zu überblickenden Institutionen den aus dem Demokratieprinzip abgeleiteten, zwingenden Organisationsregeln entsprechen. Insbesondere bei plural aus Staatsvertretern und Repräsentanten sonstiger öffentlicher oder privater Interessen zusammengesetzten Gremien ist dann für die Ausübung von "Staatsgewalt in der Form amtlichen Handelns mit Entscheidungscharakter ${ }^{14}$ unter Legitimationsgesichtspunkten zusätzlich zum Beschluß der Mehrheit der Mitglieder noch ein zustimmender Mehrheitsbeschluß der personell legitimierten Amtsträger erforderlich (sog. Prinzip der »doppelten Mehrheit «). Das BVerwG deutet in seinen Vorlagebeschlüssen zwar an, daß von diesen hohen personellen Legitimationsanforderungen möglicherweise die berufsständischen Selbstverwaltungskörperschaften wie die Kammern und Innungen ausgenommen werden könnten, weil sich deren Aufgaben, Organisation und Befugnisse auf die eigenen Angelegenheiten der in ihnen zusammengefaßten Rechtssubjekte beschränkten." Allerdings ist sich das Gericht schon über die denkbaren Begründungen einer solchen Ausnahme im unklaren. Es schwankt zwischen der These, die fehlende individuelle Legitimation der Amtswalter könne hier eventuell substituiert werden durch eine sachlich-inhaltliche Legitimation, die durch die Detailliertheit und Klarheit der gesetzlichen Vorgaben sowie behördliche Aufsichts- und Weisungsrechte gestiftet werde, ${ }^{16}$ und der Idee, daß u. U. die Mitglieder dieser berufsständischen Körperschaften als legitimationsfähiges "Teilvolk « i.S. von Art. 28 Abs. I GG angesehen werden könnten. ${ }^{17}$

Aber nicht nur die gesetzlichen Grundlagen von Kammern und Innungen sowie Wasserwirtschaftsverbänden stellen die Vorlagebeschlüsse des 6. Senats auf den Prüfstand dieses Modells der demokratischen Vernunft. Vielmehr gilt dies in gleicher Weise für alle öffentlich-rechtlich organisierten Unternehmungen (wie die öffentlichrechtlichen Versicherungen und Brandkassen, die Sparkassen und Landesbanken, die

14 Stchende Forme! seit BVerfGE 83, 60ff., 73; BVerfGE 93, 37 ff., 67; BVerwG, Vorlagebeschlüsse v. 17. 12.1997, Az: $6 \mathrm{C}$ t und 2.97, Umdruck 6 C 2.97 S. 26.

Is EmschGG, Umdruck 6 C 2.97 S. 29.

16 EmschGG, Umdruck 6 C 2.97 S. 29

17 EmschGG, Umdruck 6 C 2.97 S. 23 
Vielfalt der Eigenbetriebe, die Deichverbände etc.), in deren Organen neben den Arbeitnehmervertretern häufig insbesondere Repräsentanten wirtschaftlicher Interessen oder althergebrachter ständischer Traditionen Sitz und Stimme haben, aber auch für den Gesamtbereich der »Staatskulturverwaltung " (von Staatstheatern und Opern über die Filmförderung und -bewertung bis hin etwa zu den niedersächsischen "Landschaften «), für die staatsfernen, abcr staatlich organisierten Bereiche des Wissenschafts- und Forschungssektors, für die öffentlich-rechtlichen Funk- und Fernsehanstalten, die Sozialversicherungsträger und nicht zuletzt für die sog. "ministerialfreien Räume " weisungsunabhängiger Institutionen (wie die Bundesbank, das Bundeskartellamt, die Rechnungshöfe etc.). Es geht damit um nichts weniger als die Durchforstung des gesamten Verwaltungsrechts und der gesamten Verwaltungspraxis der Bundesrepublik und ihre u. U. gebotene Neuorganisation - eine Neuorganisation, deren Notwendigkeit lediglich unter einem einzigen Gesichtspunkt verhandelt wird, nämlich demjenigen der demokratischen Legitimation in der vom Zweiten Senat des BVerfG vertretenen etatistischen Version. Dies verleiht den durch die Vorlagebcschlüsse des BVerwG angestoßenen Verfahren vor dem BVerfG eine Bedeutung, die kaum größer eingeschätzt werden kann.

Ob der Zweite Senat des BVerfG die Gelegenheit ergreift, sich mit diesen Konsequenzen seines Demokratiekonzepts auseinanderzusetzen, die ihm durch die Beschlüsse des BVerwG in aller Deutlichkeit vor Auge geführt worden sind, steht zur Stunde noch nicht fest. Es wäre zu wünschen, daß diese Richtervorlage nicht als unzulässig zurückgewiesen wird. Denn die Revision des zum Verfassungsdogma erstarrten »Böckenförde-Modells « der Demokratie ist im Intercssc der Wicdergcwinnung der Offenheit des demokratischen Prozesses selbst, darin ist sich ein beachtlicher Teil der rechtswissenschaftlichen Literatur ${ }^{18}$ einig, dringend erforderlich.

\section{Volkssouveränität, demokratische Legitimation der Staatsgewalt und Verwaltungsorganisation}

Die Kernaussagen der verfassungsgerichtlichen Lesart des Demokratieprinzips verdanken ihre vordergründige Plausibilität nicht zuletzt dem Umstand, daß es sich um einprägsame Formeln handelt, die gebersmühlenartig beständig wiederholt werden. Dies verleiht ihnen einen beträchtlichen suggestiven Effekt. Die nachstehenden Ausführungen nehmen dieses demokratietheoretische Legitimationsmodell näher unter die Lupe. Dabei werden die zentralen Passagen abschnittsweise dargestellt und auf ihren normativen wie empirischen Gehalt hin befragt. Auf diese Weise wird erstens ihr eigentümliches Changieren zwischen normativen und deskriptiven Aussagen, Legitinations- und Steuerungsfragen und damit Gerechtigkeits- und Machtgesichtspunkten sichtbar. Zweitens tritt der spezifisch deutsche, sowohl volksbezogene wie etatistische Traditionszusammenhang deutlicher hervor, in dem diese Demokratieauffassung wurzelt. Und drittens wird herausgearbeitet, daß dieses demokratische

$18 \mathrm{Vgl}$. zur Darstellung und Kritik insbes. Schuppert, Verfassungsrecht und Verwaltungsorganisation, Der Staat 1993, 581 ff.; Bryde, Die bundesrcpublikanische Volksdemokratie als Irrweg der Demokratietheorie, in: Staatswissenschaften und Staatspraxis, 1994, S. 305 ff.; Dopatka, Mitbestimmung im öffentlichen Dienst revisited. Konservativer roll back uder Angleichung der Verhaltnisse?, KJ 1996, 224 ff.; Plander, Personalvertretungen als Grundrechtshilfe im demokratischen und sozialen Rechtsstaat, 1995; Rinken, Demokratie und Hicrarchie. Zum Demokraticverständnis des Z.weiten Senats des Bundesverfassungsgerichts, KritV 1996, 282 ff.; Blanke, Verwaltungsmodernisierung. Direktionsreche des Arbeitgebers, Mitwirkungsrechte des Personalrats und Tarifautonomie, 1998, S. $64 \mathrm{ff} ., 73 \mathrm{ff}$. 
Legitimationskonzept auch insoweit in die Irre führt, wie aus ihm verbindliche Organisationskonzepte für die Verwaltung abgeleitet werden.

Die Kritik an diesem Demokratiemodell des Zweiten Senats des BVerfG zielt darauf, das konstitutionelle Rangverhältnis zwischen dem parlamentarischen Gesetzgeber und der Verwaltung wieder ins demokratische Lot zu rücken. Aus dem Demokratiegebot des Grundgesetzes folgt nicht etwa eine zwingende Struktur der Verwaltungsorganisation, die dem Gesetzgeber vorgegeben ist. Vielmehr ist im Gegenteil der Gesetzgeber durch das Demokratieprinzip ermächtigt, nicht nur die Inhalte öffentlicher Aufgaben festzulegen, sondern auch über die Formen ihrer Erledigung weitgehend frei zu entscheiden. Es geht also darum, demokratische Gestaltungsspielräume des Parlaments gegenüber der Verwaltung zurückzugewinnen, die die herrschende Lesart des Demokratieprinzips systematisch verstellt hat. Damit soll nicht etwa im Gegenzug die These aufgestellt werden, daß die Verfassung ein alternatives Organisationsmodell der Verwaltung vorschreibe. Wieviel Beschäftigtenmitbestimmung in welchen Angelegenheiten und in welcher Form erlaubt oder gar geboten ist, legt das Demokratieprinzip der Verfassung sowenig fest wie die Frage, ob und in welchem Umfang Privatrechtssubjekte bei der Erledigung von Aufgaben, die im öffentlichen Interesse liegen, mit herangezogen werden können.

\section{Alle Staatsgewalt geht vom Volke aus}

Ausgangspunkt der Argumentation ist Art. 20 Abs. 2 GG. Dort heißt es: "Alle Staatsgewalt geht vom Volke aus. Sie wird vom Volke in Wahlen und Abstimmungen und durch besondere Organe der Gesetzgebung, der vollziehenden Gewalt und der Rechtsprechung ausgeübt. «Der Grundsatz, daß alle Staatsgewalt vom Volk ausgehen muß, statuiert das Prinzip der Volkssouveränität. Vermittelt über die Art. 1 Abs. I, 79 Abs. ${ }_{3}$ GG ist es in den Rang einer "Ewigkeitsgarantie" erhoben. Gem. Art. 28 Abs. I GG gilt es auch für die Gebietskörperschaften der Länder, Kreise und Gemeinden.

Der Grundsatz der Volkssouveränität, so heißt es zu Beginn der Darstellung des Demokratieprinzips im Beschluß des BVerfG v. 24. 5 . 1995 , $^{19}$ »legt fest, daß das Volk die Staatsgewalt, deren Träger es ist, außer durch Wahlen und Abscimmungen durch besondere Organe der Gesetzgebung, der vollziehenden Gewalt und der Rechtsprechung ausübt. Das setzt voraus, daß das Volk einen effektiven Einfluß auf die Ausübung der Staatsgewalt durch diese Organe hat. Deren Akte müssen sich auf den Willen des Volkes zurückführen lassen und ihm gegenüber verantwortet werden. Dieser Zurechnungszusammenhang zwischen Volk und staatlicher Herrschaft wird vor allem durch die Wahl des Parlaments, durch die von ihm beschlossenen Gesetze als Maßstab der vollziehenden Gewalt, durch den parlamentarischen Einfluß auf die Politik der Regierung sowie durch die grundsätzliche Weisungsgebundenheit der Verwaltung gegenüber der Regierung hergestellt.«

Diese Formulierungen beginnen im Ausgangssatz mit dem anspruchsvollen normativen Programm der klassischen Demokratievorstellungen. Dieses wird jedoch nicht etwa näher erläutert, sondern sogleich empiristisch umgedeutet und normativ entwertet. Denn von diesem Ausgangspunkt - das Volk ist Träger der Staatsgewalt und übt sie aus - bleibt schon im Folgesatz, verbunden durch ein harmloses "das setzt voraus«, lediglich das Erfordernis eines neffektiven Einflusses« auf die Ausübung der Staatsgewalt übrig. Damit ist dem normativen Anspruch der Demokratie, self-government zu sein, bereits der Stachel gezogen.

Es ist notwendig, in diesem Zusammenhang kurz an die Programmatik der Demo-

19 BVerfGE 93, 37 ff., 66 . 
kratie zu erinnern: Demokratie heißt wörtlich Volksherrschaft, Selbstgesetzgebung, Selbstregierung und Selbstverwaltung des Volkes. Die mit dieser Idee verbundene Intuition einer hypothetischen, als regulative Idee oder "Prüfstein der Vernunft" fungierenden Identität von Regierenden und Regierten, Befehlenden und Gehorchenden, Adressaten und Autoren der Gesetzgebung ist Gemeingut nahezu aller Demokratietheorien und inspiriert bis heute die Debatcen um Inhalt und Reichweite des Demokratieprinzips. ${ }^{20}$ Volkssouveränität ist in dieser Tradition nicht die machtstaatlich entfesselte Souveränicät eines Kollektivverbandes, sondern Resultat wechselseitiger Anerkennung der zum Gemeinwesen verbundenen Bürger als moralische, vernünftige Personen und der "gleichursprünglichen" Garantie des Selbstbestimmungsrechts, der Autonomie jedes Einzelnen. ${ }^{21}$ Volkssouveränität gründet im Universalismus der Menschenwürde. Die »normativ orientierte Demokratietheorie geht von der Würde des Menschen aus «. ${ }^{22}$ "Verfassungsrechtliche Grundnorm für die demokratische Ordnung des Grundgesetzes ist nicht nur Art. 20 Abs. 2 GG, sondern auch die auf Selbstbestimmung drängende Fundamentalnorm des Art. x Abs. I GG. 'Demokratie ist die organisatorische Konsequenz der Menschenwürde «. ${ }^{2}$

Die demokratische Frage, welche Strukturen in Staat und Gesellschafe, Politik und Wirtschaft, Kultur, Bildung und Erziehung, Familie und Lebenswelt dieser Idee einer Selbstregierung am ehesten entsprechen, stellt sich für den Zweiten Senat des BVerfG aber schon mit dem zweiten Satz seiner Darstellung des Demokratieprinzips nicht mehr. Damit wird die soziale Dimension des Demokratiebegriffs ausgeblendet, mit der der SPD-Politiker Carlo Schmid im r. Deutschen Bundestag 1949 die sozialdemokratische Lesart des Demokratiekonzepts des GG umrissen hat: „Demokratie ist nur dort eine lebendige Wirklichkeit, wo man bereit ist, die sozialen und ökonomischen Konsequenzen aus ihren Postulaten zu ziehen ... Dazu gehör, daß man den Menschen herausnimmt aus der bloßen Objektsituation - nicht nur im formalpolitischen Bereich, sondern auch und gerade dort, wo der Schwerpunkt seines Lebens liegt, nämlich im ökonomischen und sozialen Bereich «. ${ }^{24}$ Derartige Demokracisierungsvorstellungen finden im Konzept der Demokratietheorie des Zweiten Senats des $B V e r f G^{2 s}$ keinen Raum. Stact dessen spannt sich gleich zu Beginn ein bipolares Gefüge auf: Hier das Volk, dort der Staat. Demokratie ist nur mehr eine Frage des effekciven Einflusses des Volkes auf den Staat (und taugt nicht für die Gesellschaft). Der Ruf „wir sind das Volk « ist offenbar nicht bis Karlsruhe vorgedrungen.

Unterstellen wir einstweilen, ein in dieser Weise reduzierter normativer Anspruch an das Konzept der Demokratie nach dem GG sei gleichwohl zu akzeptieren. Wodurch wird dann, so ist zu fragen, dieser »effektive Einfluß « des Volkes auf das Handeln der staatlichen Organe gewährleistet? Dies geschieht nach Meinung des Zweiten Senats

20 Schon Samuel Pufendorf (1632-1694) definiert Demokratie als Gesellschaftsordnung »wo derjenige, der befiehlt und derjenige, der gehorcht, derselbe ist $«$, zit nach Neumann, Demokratietheorien-Modelic zur Herrschaft des Volkes, iл: ders. (Hrsg.), Handbuch Politische Theorien und Ideologien, Bd. 1, 2. Aufl. r998, S. 60; zur neueren Debatte vgl. Rodel/Frankenberg/Dubiel, Die demokratische Frage, 1989; Habermas, Fakrizität und Geltung, 1992; ders., Drei normative Modelle der Demokratie, in: ders., Die Einbeziehung des Anderen, 1996; zur Diskussion um dic in dem Band "Fakrizität und Geltung* entalcete Rechtstheorie von Habermas vgl. die Beitrage von Blanke, Kutscha und Gunther, in: KJ 1994, 439 ff.

21 Ausführlich hierzu: Habermas, Fakcizität und Geltung, 1992; Honneth, Kampf um Anerkennung. Zur moralischen Grammatik sozialer Konflikte, 1994; Blanke, Die praktische Bedeutung der kommunikativen Rechtstheorie oder: Der Preis des Rechtsfortschritts, in: Buckmiller/Perels, Opposition als Triebkraft der Demokratic, FS fir Jürgen Seifert, I998, S. 33 ff.

22 Neumann (Fn. 20), S. is; vgl. auch Besson/Jasper, Das Leitbild der modernen Demokratie. Bauelemente einer freiheitlichen Staatsordnung, 1990, S. 13 .

23 Rinken (Fn. 18), S. 28 fff., 295 unter Verwendung eines Zitats von Häberle, Die europäische Verfassungsstaatichkeit, KritV ig95, S. $298 \mathrm{ff} ., 303$.

${ }_{24}$ BT-Pror. d. 10. Sitzung v. 29.9.1949.

2) BVerfGE 93, 37 ff., 66. 
des $B$ Verf $G^{26}$ dadurch, daß sich deren Akte »auf den Willen des Volkes zurückführen« lassen und ihm gegenüber "verantwortet " werden. Diesen "Zurechnungszusammenhang* stellen "vor allem» die Parlamentswahl, das Gesetzgebungsprogramm als "Maßstab der vollziehenden Gewalt «, der "parlamentarische Einfluß « auf die Politik der Regierung sowie die "grundsätzliche Weisungsgebundenheit« der Verwaltung gegenüber der Regierung her. Die Aussage, daß sich durch die beschriebenen $\mathrm{Me}$ chanismen die Akte der Staatsorgane auf den Willen des Volkes zurückführen ließen, entbehrt allerdings bei genauerer Betrachtung jeder empirischen Realität. Dies erschließt sich schon durch die schlichte Frage, wie denn die identifizierende »Rückführung " des sich in einer unabsehbaren Fülle gleichzeitiger Verwaltungsentscheidungen artikulierenden Staatswillens auf den einmal, nämlich im punktuellen Wahlakt des Parlaments artikulierten Volkswillen auch nur festgestellt, geschweige denn gesichert werden kann. Als empirisches Projekt würde dies auf ein monströses, notwendig zum Scheitern verurteiltes Unterfangen hinauslaufen. Dies liegt schon deswegen auf der Hand, weil niemand in der Lage ist, diese Äußerungen des Staatswillens auch nur zu registrieren und zu überblicken, um sodann ihre Summe zu ziehen und diese mit dem gänzlich undifferenzierten Volkswillen der (welcher?) Parlamentswahl zu vergleichen. Die Rede vom »Volkswillen« ist überdies irreführend und zudem, da das »Volk in dieser Formulierung als »real existierendes Megasubjekt « gedacht wird, metaphorisch. Konkret handelt es sich idealiter nicht einmal um die politische Quersumme aus den Willensbildungsprozessen aller Staatsbürger, sondern allenfalls um den Parteien- und Regierungswillen, der sich in den Verästelungen der staatlichen Entscheidungen manifestiert. Ohne die dominante Rolle der (weitgehend selbst vverstaatlichten<) politischen Parteien bei der Artikulation des politischen Willens der Bürger, der Parlamentswahl, der Regierungsbildung und Gesetzgebung läßt sich die Praxis des demokratischen Prozesses in den modernen Massendemokratien nicht beschreiben. ${ }^{27}$ Ebenso kann weder im Blick auf die Fülle der weisungs- und unterrichtungsfreien Räume in der Verwaltung ${ }^{28}$ von deren "grundsätzlicher Weisungsgebundenheit « noch im Blick auf normativ weitgehend ungesteuerte - und auch nicht steuerbare - Aufgaben der Verwaltung etwa im weiten Bercich der Planung und der sog. Risikoverwaltung ${ }^{29}$ pauschal vom Gesetz "als Maßstab der vollziehenden Gewalt « gesprochen werden.

Die »Rückführung « der Akte der Staatsorgane auf den Volkswillen und die Garantie eines entsprechenden "Zurechnungszusammenhangs zwischen Volk und staatlicher Herrschaft « durch die genannten Mechanismen sind demnach keineswegs Beschreibungen einer material gehaltvollen Relation, die einer empirischen Überprüfung zugänglich wären. Es geht aber andererseits auch nicht um normative Aussagen, die im Hinblick auf ihre Gerechtigkeitsannahmen kritisch hinterfragt werden könnten. Vielmehr handelt es sich um metaphorische Beteuerungen der fälschlich als "effektiver Einfluß deklarierten legitimierenden Effekte und Leistungen, die die im GG vorgesehenen Prozeduren parlamentarischer Wahlen im Verein mit einigen rechtsstaatlichen Grundsätzen für die Staatstätigkeit erbringen sollen. Diese Redeweise vermengt den Unterschied von empirischen (»effektiver Einfluß«) und normativen Aussagen ("Verantwortung " gegenüber dem Volkswillen, "Zurechnungszusam-

26 BVerfGE 93, $37 \mathrm{ff} ., 66$.

27 Bereits in einer der ersten Entscheidungen des BVerfG findet sich die inzwischen wieder in Vergessenheit

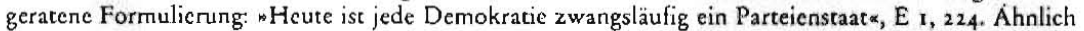
BVerfGE 2 ff., I r: $\times$ Der Volkswille kann ... in der Wirklichkcit des modernen großen Staates nur in den Parteien als politischen Handlungseinheiter erscheinen $\alpha$.

$28 \mathrm{Vgl}$. die gleichnarnige Habilitationsschrift von Ocbbecke, 1986.

29 Hierzu ausfuhrlieh v. Danwitz, Der Grundsatz funktionsgerechter Organisationsstruktur, Der Staat, 1996, 329 lf., $338 \mathrm{ff}$ 
menhang «) und verwechselt Machtfagen mit Legitimationsfragen. Diesem unklaren Status ihrer Sätze entspricht ihr deklaratorischer Charakter, der sie jeder diskursiven Infragestellung entzieht und in Gefahr ist, sich zu einem Dogmatismus zu entwikkeln. ${ }^{\text {jo }}$

\section{Das nationalstaatszentrierte, kollektiv-identitäre Demokratiekonzept}

Bemerkenswerterweise ist in der durch die Rechtsprechung ${ }^{31}$ und gewichtige Teile der staatsrechtlichen Literatur ${ }^{32}$ kanonisierten deutschen Demokratietheorie von dem politisch wichtigsten und effektivsten Mittel zur Sicherung des demokratischen Einflusses auf die Staatsgewalt, nämlich dem Gebrauch der politischen Kommunikationsfreiheiten - insbesondere der Meinungs-, Presse-, Rundfunk-, Versammlungsund Demonstrationsfreiheit - und der Drohung mit dem Verlust der politischen Macht durch Abwahl der Regierung, sei es im Zuge der regelmäßigen Parlamentswahlen, sei es bereits zuvor durch parlamentarisches Mißtrauensvotum, nicht die Rede. Nicht die Kontrolle der Staatsgewalt durch den demokratischen Prozeß, die diskursive politische Meinungs- und Willensbildung der Zivilgesellschaft, die demokratische Funktion der parlamentarischen und außerparlamentarischen Opposition und die Rhythmik der Wahlen sind Thema dieser judikativen Demokratietheorie, sondern genau umgekehrt: Es geht ihr um die Sicherung der Effektivität staatlicher Machtausübung, nachdem die Regierung einmal das Mandat hierzu in den Parlamentswahlen erhalten hat. Wie kann gewährleistet werden, so fragt sie, daß der vom Volk ursprünglich in den Wahlen ausgegangene Wille sich durch die Kapillaren des vielmaschigen Netzwerks der Verwaltungstätigkeit hindurch unverfälscht und idenrisch erhält und sich als Staatswille effektiv durchsetzt?

Dieses Demokratiekonzept widerspricht dem selbstyerwaltungsfreundlichen, partizipativen Demokratieverständnis des Grundgesetzes. Das Grundgesetz hat der Idee einer allein auf den parlamentarischen Wahlakr fixierten Demokratiekonzeption eine deutliche Absage erteilt: Deshalb enthält es sowohl individuelle wie kollektive politische Grundrechte (Art.s Abs. I S. I, Art. 8 Abs. I, Art.9 Abs. I und 3 GG), garantiert die Freiheit der Berichterstattung durch Presse, Rundfunk und Film (Art. 5 Abs. I S. 2 und 3 GG), die Wissenschafts- und Forschungsfreiheit (Art. s Abs. 3 GG) und räumt den politischen Parteien eine besonderc Stellung bei der 》Vorformung « des demokratischen Willensbildungsprozesses ein (Art. 2 I Abs. I GG). Außerdem ist der Zentralismus der Staatsgewalt durch die Verbürgung des föderalen Staatsaufbaus sowie die Selbstverwaltungsautonomie der Gebietskörperschaften (Art. 28 GG) ge-

30 Rinken komment:ert den Argumentationsstil der Entscheidung des Zweiten Senats v. 24. 5. 1995, BVcrfGE 93, 6o ff. treffend wie folgt: "Dic Entschcidung ... argumentiert nicht, sic dekrctiert; sic deduzicrt ihre Ergebnisse nicht aus der Verfassung, sondern aus vorverfassungsmaßigen Pramissen; sie legt diese Pramissen nicht offen, sondern setzt sie voraus*, KritV 1996, S. 288.

31 Insbesondere durch die Entscheidungen der hessischen, nordrhein-westfälichen und rheinland-pfalzischen Staatsgerichtshofe, HessStGH 30. 4. 1986, PersR 1986, 148 ff.; VerfGH NW is. 9. 1986, DVBl. 1986, $1196 \mathrm{ff}$; VerfGH RP 18.4.1994, PersR 1994, $272 \mathrm{ff}$.

32 Vgl. aus der Debatte *Personalvertretung und Dumokratiegcbot « nur Isensec, Offentlicher Dienst, in: Benda/Maihofer/Vogcl (Hrsg.), Handbuch des Verfassungsrechts der Bundesrepublik Deutschland, 2. Aufl. 1994, S. I $527 \mathrm{ff}$; Kisker, Grenzen der Mitbestimmung im öffenclichen Dienst, 1984; ders., Ein Grundrecht auf Teilhabe an Herrschaft?, in: Festschrift fuir Willi Geiger, 1989, S. 234 ff.; ders., Mitbestimmung total. Zur Neuordnung des Personalvertretungsrechts in Schleswig-Holstein, PersV r g92, $1 \mathrm{ff}$; H. H. Klein, Die Rechtsprechung des Bundesverfassungsgerichts zum Personalvertretungsrecht, PersV 1990, 49 ff.; Ossenbuhl, Grenzen der Mitbestimmung im öffentlichen Dienst, 1986; Puttner, Mitbestimmung und Mitwirkung des Personals in der Verwaltung, in: H.-J. Oertzen (Hrsg.), Demokratisierunge und Funktionsfähigkeit der Verwaltung, 1974, S. 73 ff.; Schenke, Zur Verfassungswidrigkeit des SchleswigHolsteinischen Gesetzes uber die Mitbestimmung der Personalrate vom rı. 1 2. 1990, PersV r992, 2.89ff.; ders., Personalvertretungsrecht und Verfassung, JZ i $99 \mathrm{r}, 58 \mathrm{Iff}$. 
brochen. Damit öffnet das Grundgesetz das zentralistische, nationalstaatliche Demokratiemodell »nach unten« hin zur demokratischen Selbstverwaltung der Staatsbürger. Gleiches gilt gem. der in Art. ${ }_{24}$ Abs. I GG enthaltenen Ermächtigung zur Übertragung von Hoheitsrechten auch »nach oben« hin in Richtung auf supranationale Instanzen wie die Europäische Union.

Demgegenüber unterstellt die in Teilen der Staatslehre und der Verfassungsjudikatur des Zweiten Senats des BVerfG dominierende Demokratiekonzeption eine fiktive vorstaatliche Einheit und Homogenität des deutschen Volkes. Dieses bildet gewissermaßen die natürliche Substanz der deutschen Demokratie. Damit ebnet dieser Typus einer kollektiv-identitären Demokratiekonzeption die kulturellen, religiösen, ethnischen, sozialen und politischen Differenzen in einer modernen pluralistischen Gesellschaft ein zugunsten einer durch »Blutsbande» definierten Einheitsidee des »deutschen Volkes«. Diese Lesart des Demokratieprinzips geht nicht zufällig auf die kollektiv-identitätstheoretische Staatstheorie von Carl Schmitt aus der Weimarer Zeit zurück, ${ }^{33}$ die sich unschwer dem NS-System und seinem rassistischen Legitimationskonzept anverwandeln konnte: "Der zentrale Begriff der Demokratie ist Volk, nicht Menschheit. Es gibt, wenn Demokratie überhaupt eine politische Form sein soll, nur eine Volks- und keine Menschheitsdemokratie «. ${ }^{44}$ Ganz in der Tradition dieser Entgegensetzung von einheitstiftender, völkischer Homogenität gegen »eine humanistisch konzipierte >Menschheit<, mit der sich der moralische Begiff des gleichmäßigen Respekts für jedermann verbindet ", "ss besteht Böckenförde darauf, daß es im Gegenzug zu den Prozessen der Individualisierung "einer relativen Homogenisierung in einer gemeinsamen Kultur (bedarf), ... damit die tendenziell atomisierte Gesellschaft wieder zusammengehalten und - ungeachtet ihrer differenzierten Vielfalt - zur handlungsfähigen Einheit verbunden wird. In diese Funktion tritt neben und nach der Religion die Nation und das ihr zugehörige nationale Bewußrsein ... So kann es nicht das Ziel sein, nationale Identität zu überholen und zu ersetzen, aucb nicht zugunsten eines menschenrechtlichen Universalismus «. ${ }^{36}$ Der Siegeszug dieses "volksdemokratischen " ${ }^{37}$ Konzepts hat in der verfassungsgerichtlichen Rechtsprechung $^{3^{8}}$ konsequent dazu geführt, daß die gesetzliche Einräumung eines an eng gefaßte Voraussetzungen gebundenen Ausländerwahlrechts zu nur lokal begrenzten und bedeutsamen politischen Gremien für verfassungswidrig erklärt worden ist - eine Rechtsauffassung, deren Konsequenzen inzwischen durch die Einräumung des Wahlrcchts für EG-Ausländer zu den Gemeinden und Kreisen in Art. 28 Abs. I S. 3 GG im Zuge der "Maastricht-Novelle» des GG v. 2 I. I 2. 1992 entsprechend Art. 8b Abs. I EWG-Vertrag korrigiert worden sind.

\section{Der weite Begriff der Staatsgewalt und ihrer Ausübung}

Diesem Demokratiemodell zufolge muß alles staatliche Handeln demokratisch legitimiert sein, wobei der Begriff der Staatsgewalt weit gefaßt wird: "Ausübung von 'Staatsgewalt< beschränkt sich nicht auf den Bereich hoheitlichen Handelns, bei dem

33 Zur ${ }^{2}$ Fortentwicklung und Veredelung des Demokraticbegriffs von Carl Schmitt insbesondere durch E.-W. Böckenförde« resumiert Emde, Die demokratische Legitimation der funktionalen Selbstverwaltung, 199x, in seiner detailgenauen Rekonstrukcion: "So subuil und feinsinnig sich dieses Verfahren ausnimmt - auf die geltende Verfassung kann es sich nicht berufen $\alpha$. S. 4 ०1.

34 Verfassungslehre $(1928), 1983$, S. 234.

3s Habermas, Inklusion - Einbeziehen oder Einschließen? Zum Verhälenis von Nation, Rechtsstaat und Demokratie, in: ders., Dic Einbeziehung des Anderen (F'n. 20), S. 154 ff., 162.

36 Die Nation, in: FAZ v. 30.9. 1995.

37 Bryde (Fn. 18 ).

38 BVerfGE 83, $37 \mathrm{ff}$. (Kommunalwahirecht Schleswig-Holstein) und E 83, 60 ff. (Kommunale Bezirksversammlungen Hamburg). 
der Staat seine einseitige hoheitliche Überlegenheit in den Formen des öffentlichen

Rechts zur Geltung bringt, sie umfaßt die Wahrnehmung der dem Staat zukommenden bzw. von ihm tatsächlich in Anspruch genommenen Aufgaben insgesamt «. ${ }^{39}$ Innerhalb dieses weiten Rahmens, der auch das privatrechtliche Handeln der öffentlichen Hand und die Tätigkeit von Privatrechtssubjekten, die mit öffentlichen Aufgaben "beliehen « sind, umfaßt, bedeutet das Ausüben von Staatsgewalt in den Worten des Zweiten Senats des BVerfG: »Als Ausübung von Staatsgewalt, die demokratischer Legitimation bedarf, stellt sich jedenfalls alles amtliche Handeln mit Entscheidungscharakter dar. Es kommt nicht darauf an, ob es unmittelbar nach außen wirkt oder nur behördenintern die Voraussetzungen für die Wahrnehmungen der Amtsaufgaben schaff « $^{40}$

\section{Demokratischer Einfluß und Kontrolle der Staatsgewalt: Die Hierarchie der Legitimationsquellen}

Der erforderliche "Zurechnungszusammenhang zwischen Volk und staatlicher Herrschaft « " ${ }^{41}$ wird in diesem Demokratiekonzept neben dem bereits dargestellten pauschalen Verweis auf die Parlamentswahlen, den Gesetzesvorbehalt etc. konkreter hergestell durch das Postulat unterschiedlicher Formen demokratischer Legitimation. Im Anschluß an die angeführten Eingangssätze zum Demokratieprinzip führt der Zweite Senat des BVerfG ${ }^{42}$ hierzu aus: "Für die Beurteilung, ob dabei ein hinreichender Gehalt an demokratischer Legitimation erreicht wird, haben die in der Rechtsprechung des Bundesverfassungsgerichts und in der Literatur ${ }^{43}$ unterschiedenen Formen der institutionellen, funktionellen, sachlich-inhaltlichen und der personellen Legitimation Bedeutung nicht je für sich, sondern nur in ihrem Zusammenwirken. Aus verfassungsrechtlicher Sicht entscheidend ist nicht die Form der demokratischen Legitimation staatlichen Handelns, sondern deren Effektivität; notwendig ist ein bestimmtes Legitimationsniveau. Dieses kann bei den verschiedenen Erscheinungsformen von Staatsgewalt im allgemeinen und der vollziehenden Gewalt im besonderen unterschiedlich ausgestaltet sein.“

Die Pluralisierung der Legitimationsformen macht es erforderlich, sie zueinander ins Verbältnis zu setzen und Kriterien dafür zu entwickeln, ob und inwieweit sie in der Lage sind, einander zu ergänzen, abzustützen und zu substituieren. Es entsteht die Notwendigkeit, dieses Pantheon divergierender Legitimationsquellen hierarchisch zu ordnen. Der Beschluß des BVerfG v. 24. 5. $1995^{44}$ ist insoweit im Ergebnis zwar eindeutig, in der Begründung aber wenig ergiebig. Die Aussage, es komme weniger auf die Form der Legitimation als auf ihre Effektivität, das "Legitimationsniveau « an, welches freilich von Staatsgewalt zu Staatsgewalt und selbst innerhalb ein und derselben Gewalt unterschiedlich sein könne, sind derart unpräzise, daß sie jedes Ergebnis tragen. Die auf "Organe und Amtswalter im Bereich der Exekutive bezogene Aussage, hier sei die »Ausübung von Staatsgewalt demokratisch legitimiert, wenn sich die Bestellung der Amtsträger - personelle Legitimation vermittelnd - auf das Staatsvolk zurückführen läßt und das Handeln der Amtsträger selbst eine aus-

\footnotetext{
39 Bockenförde, Demokratie als Verfassungsprinzip, in: Isensee/Kirchhoff (Fn. 1), Rn. 12.

40 BVerfGE 93, $37 \mathrm{ff}, 68$.

41 BVerfGE 93,37 ff., 66.

42 BVerfGE 93, 37 ff., 661 .

43 Hier fe.hlt im Beschlußv. 25. 5. x 995, E 93, 37 ff., 66, jeglicher Hinweis. An der entsprechenden Passage der Senatsentscheidung zum Ausländerwahlrecht in Hamburg v. 31. 10. 1990, E 83,60 ff., 72, wird aus der Literarur lediglich verwiesen auf Böckenförde, Demokratie als Verfassungsprinzip, in: Isensee/Kirchhoff, Handbuch des Staatsrechts, Bd. I, 1987, \$22 Rn. 14

44 BVerfGE 93, 37 ff., $66 \mathrm{ff}$.
} 
reichende sachlich-inhaltliche Legitimation erfährt", durch die ohne jede Erläuterung nurmehr lediglich zwei der genannten vier Legitimationsquellen aufgeführt wcrden, wird denn auch wie im delphischen Orakel schlicht dekretiert. Ebensowenig wird begründet, warum von diesen beiden Legitimationsquellen nur eine, nämlich die personelle Legitimation im Hinblick auf das Erfordernis der »ununterbrochenen Legitimationskette « näher operationalisiert wird und schließlich als ausschlaggebendes Kriterium der demokratischen Legitimation allen "amtlichen Handelns mit Entscheidungscharakter « übrigbleibt.

Die Vorlagebeschlüsse des BVerwG ${ }^{4 s}$ zeigen insoweit wenigstens im Ansatz mehr Problembewußtsein und argumentativen Begründungsaufwand. Wenn es darum geht, Rang- und wechselseitige Ergänzungs- und Substitutionsverhältnisse zwischen den unterschiedlichen Legitimationsformen zu begründen, dann muß in einem ersten Schritt der $Z_{\text {weck }}$ definiert werden, dem die Legitimation dienen soll. Zu fragen ist also: Was soll überhaupt vermittels dieser unterschiedlichen Legitimationsformen gerechtfertigt werden? Wenn dies geklärt ist dann kann im zweiten Schritt der Versuch gemacht werden, die Relevanz der Legitimationsformen im Hinblick auf diesen Zweck zu bestimmen. In direkter Anlehnung an Böckenförde ${ }^{46}$ führt das BVerwG ${ }^{47}$ hierzu in seinen Vorlagebeschlüssen aus: "Thematisch geht es immer um die Steuerung der Ausübung staatlicher Gewalt, die bei der öffentlichen Verwaltung nur durch das Zusammenwirken der personellen Legitimation der Organwalter einerseits und der sachlich-inhaltlichen Legitimation ihres gesetzesgesteuerten Handelns andererseits bewirkt werden kann. Beide Elemente der Legitimation können sich keineswegs vollständig ersetzen, wohl aber in einem gewissen Umfang substituieren, solange dadurch das geforderte Niveau an Effektivität demokratischer Legitimation nicht in Frage gestellt wird. Die Konstituierung sachlich-unabhängiger Organe erfordert etwa, daß deren organisatorisch-personelle Legitimation umfassend gewährleistet ist und auch ihr Handlungsbereich gesetzlich geregelt und umgrenzt ist. Umgekehrt mag eine strikte sachlich-inhaltliche Legitimation durch inhaltlich bestimmte gesetzliche Normierungen des Handelns und hinzutretende Aufsichtsbefugnisse demokratisch legitimierter Instanzen es ermöglichen, gesellschaftliche Gruppen punktuell an der Besetzung der Organe bzw. Auswahl der Amtswalter zu beteiligen«. Zweck der demokratischen Legitimation ist danach die Steuerung der Ausübung staatlicher Gewalt, zentrale Legitimationsformen hierfür sind die personelle und die inhaltliche Legitimation. Damit wird deutlich, daß wiederum Legitimationsfragen, die auf Rechtfertigungen, also moralische Fragen zielen, in irriger Weise gleichgesetzt werden mit Machtfragen, die empirische Sachverhalte wie etwa Aspekte möglichst effizienter Steuerung zum Gegenstand haben. Lediglich bei letzteren läßt sich nämlich eine graduelle Abstufung nach Effektivitäts- oder Niveaukriterien vornehmen. Demgegenüber bewähren sich Legitimationen, normative Rechtfertigungen wie moralische Urteile generell nach einem "Alles oder Nichts-Schema : Entweder sie tragen oder sie versagen, entweder sind sie wahr oder falsch. ${ }^{8}$ Ein bißchen Gottesgnadentum reicht ebensowenig zur Kaiserwürde wie ein kleines »excathedra " zur zuverlässigen päpstlichen oder verfassungsgerichtlichen Wahrheitsfindung. Tertium non datur.

4 Vorlagebeschlusse v. 17. r2 r997, Az: $6 \mathrm{C}$ I und 2.97

46 In: Isensec/Kirchhoff (Fn. 43), Rn. 23.

47 Vorlagebeschlüsse des BVerwG v. 17. 12 I997, Az: 6 C I und 2.97, Umdr. S. 20 f.

48 Darin sind sich jedenfalls alle nicht relativistischen, kognitivistischen Moraltheorien einig, vgl. nur Rawls, Eine Theorie der Gerechtigkeit, 1979; das GG steht in dieser auf die Philosophie von Kant zuruckgehenden Tradition, wenn es scine moralnahen Fundanentalnormen, wie Art. 79 Abs. 3 GG zeigt, nicht zur Disposition stellt - nichr cinmal zu derjenigen des verfassungsändernden Gesetzgebers. 
Auch wenn das BVerwG die Rangordnung und die wechselseitige Substitutionsfähigkeit zwischen den beiden Legitimationsformen lediglich vage umschreibt, so wird doch auch hier soviel deurlich: Der demokratische Charakter staatlicher Entscheidungen hängt nicht in erster Linie von der sachlich-inhaltlichen Legitimation, der Detailliertheit, Präzision, sachlichen Angemessenheit und normativen Richtigkeit der gesetzlichen Vorgaben ab, die das Verwaltungshandeln steuern und dessen Entscheidungen kontrollieren sollen. Vielmehr kommt ebenso wie bereits im Beschluß des Zweiten Senats des BVerfG v. 24.5. $1995^{49}$ letztlich der personell-demokratischen Legitimation der Amtsträger, die allenfalls punktuell - und lediglich möglicherweise zugunsten der Beteiligung »gesellschaftlicher Gruppen« durchbrochen werden darf, das Privileg zu, das erforderliche Legitimationsniveau zu vermitteln.

\section{Personell-demokratische Legitimation als Mechanismus zur Verselbständi- gung der Staatsgerwalt}

In all diesen Entscheidungen und staatsrechtlichen Lehrmeinungen bleibt unausgeführt, warum letztlich der personell-demokratischen Legitimation, der "ununterbrochenen Legitimationskette « die entscheidende Rolle für die Identitätsgarantie und den notwendigen "Zurechnungszusammenhang " zwischen Volkswillen und Staatswillen zukommt, wie er sich in der Unsumme allfälliger Behördenentscheidungen, seien sie auch noch so staatsfern wie bei Rundfunkanstalten, dem öffenclichen Wissenschafts- und Forschungsbetrieb oder den Entscheidungen von Theaterintendanten und Museumsleitern oder von Ausschüssen im Bereich der staatlichen Filmförderung ausdrückt. Dies ist nicht nur angesichts der Vielfalt entgegenstehender Beispiele aus dem Verwaltungsrecht und der Verwaltungspraxis, sondern auch in der Theorie keineswegs derart selbstverständlich, daß es insoweit keiner Erläuterung bedürte. Denn nach der klassischen Konzeption der - durchaus vordemokratischen Gewaltenteilung stand zweifelsohne ganz die Idee der Programmierung der Staatstätigkeit durch die Gesetzgebung im Vordergrund. Diese im aktuell modischen Staatsrechtsjagon als "sachlich-inhaltliche Legitimation « bezeichnete Funktion sollte die Suprematie der Legislative durch die inhaltlich-kategoriale Allgemeinheit des Gesetzes einerseits, die Präzision seiner Begrifflichkeit unter Vermeidung vager Generalklauseln und Ermächtigungsnormen andererseits garantieren. Dieser Vorstellung entspricht es, wenn etwa Montesquieu dem Richter die Rolle als "Mund des Gesetzes " ("bouche de la loi«) zuschreibt, der lediglich zu verkünden habe, was vom Souverän mit dem Gesetz bereits entschieden sei, oder wenn berichtet wird, der Preußische König Friedrich der Zweite habe ein Verbot zur Kommentierung des Allgemeinen Preußischen Landrechts statuiert, um der Verfälschung des Gesetzeswillens durch eigenmächtige Interpretationen vorzubeugen. Selbst noch unmittelbar nach dem Ende der Weimarer Republik hat der Arbeitsrechtler und Rechtstheoretiker Franz Neumann ${ }^{\circ}$ die Rationalität des Gesetzes als zentrales Merkmal der »Vernünftigkeit" der Gesellschaft allein in diesen Formbestimmungen der begrifflichen Allgemeinheit und der auf ihr beruhenden Vorhersehbarkeit und Berechenbarkeit der Rechtsanwendung erblickt.

Heute kann als gesichert gelten, daß eine derart strikte konditionale Programmierung

49 BVerfGE 93, $37 \mathrm{ff}, 66 \mathrm{ff}$.

so Die Herrschaft des Gesct\%es (englisch 1936), deutsch 1980, S. 245 ff., 314 ff.; vgl. hierzu Blanke, Kirchheimer, Neumann, Preuß: Die Radikalisierung der Rechtstheorie, in: Perels (Hrsg.), Rechc, Demokracie und Kapitalismus. Akrualitat und Probleme der Theoric Franz L. Neumanns, 1984, S. 16 63 ff. sowie Intelmann, Franz L. Neumann, Chancen und Dilemma des politischen Reformismus, 1996. 
der Staatstätigkeit bereits aus begrifflich-hermeneutischen Gründen unmöglich ist." Aus rechtstheoretischer Sicht ${ }^{52}$ kann ein solches Modell der Gesetzesherrschaft allenfalls idealtypisierend einer vergangenen Epoche moderner Staatlichkeit, nämlich der 2. Hälfte des 19. Jahrhunderts, zugeschrieben werden. Politikwissenschaftliche ${ }^{31}$ wie organisationstheoretische ${ }^{54}$, gesellschafts ${ }^{-5}$ wie betriebswirtschaftliche ${ }^{56} \mathrm{Be}-$ funde haben zudem längst den Nachweis geführt, daß eine derartige Steuerungskonzeption erheblich zu undifferenziert, zu wenig komplex und deshalb nicht in der Lage ist, zu beobachten und zu thematisieren, wie Kommunikation, Strukturierung und Entscheidung in Organisationen überhaupt stattfindet.

Dies mag zwar erklären, warum gleichsam als Kompensation für diesen Verlust an legislativen Steuerungsmöglichkeiten (oder besser: Steucrungsillusionen) die personelle Legitimation ins Zentrum der verfassungsrechtlichen Aufmerksamkeit rückt. $\mathrm{Ob}$ die Erwartung jedoch gerechtfertigt ist, dadurch verlorenes Steuerungs- und Legitimationsterrain wiederzugewinnen, muß empirisch bezweifelt werden. Völlig zutreffend hat z. B. das OVG NW'7 in der Berufungsinstanz des durch die Vorlagebeschlüsse des BVerw $G$ ausgesetzten Verfahrens die tatsächlichen Effekte, die sich aus dem Postulat der »ununterbrochenen Legitimationskette ergeben, folgendermaßen charakterisiert: »Eine solche Forderung leistet für das Demokratieprinzip nichts, wo sie zum bloßen Selbstzweck wird. Der Gedanke der ununterbrochenen Legitimationskette büßt jede Aussagekraft im Hinblick auf das Demokratieprinzip ein, wenn der Legitimationsstrang des jeweiligen Amtswalters bzw. Organmitglieds zum Volk immer länger wird und je mehr der Legitimationsakt zeitlich zurückliegt. So ist schwer nachvollziehbar, warum die Amtsführung eines Bediensteten deshalb als demokratisch legitimiert anzusehen sein soll, weil ihm sein Amt vor langer Zeit von einem Dienstvorgesetzten übertragen worden ist, der zuvor von der Landesregierung ernannt worden ist, die ihrerseits von der Volksvertretung her legitimiert war. In derartigen Fällen kann sich die konkret personale Beziehung zum Volk in eine bloße Fiktion verflüchtigen, die eine reale Aussage über die demokratische Qualität des amtlichen Handelns nicht mehr erlaubt. « Im Ergebnis bewirkt damit das Primat der personell demokratischen Legitimation, wenn man es auf die Realität der überwiegend lebenszeitlichen Ernennung der beamteten wie der tariflich abgesicherten Amtswalter in der Bundesrepublik bezieht, nicht die Stärkung des »effektiven Einflusses* des Volkes auf die Verwaltungsentscheidungen, sondern im Gegenteil die Verselbständigung des Staatsapparats gegenüber dem Souverän und seinen parlamentarischen Repräsentanten.

SI Vgl. hierzu die Darstellung der «unhintergehbaren« Grenzen des Gesetzes als Steuerungsmedium beí Dreier, Hierarchische Verwaltung in demokratischen Staat, 1991, S. $164 \mathrm{ff}$.

s2 Exemplarisch sei verwiesen auf die Schemarisierung bei Teubner, Verrechtlichung - Begriffe, Merkmale, Grenzen, Auswege, in: Zacher/Simitis/Kübler/Hopt/Teubner, Verrechclichung von Wirsschaft, Arbeit und sozialer Solidarität, $198_{4}$, S. $289 \mathrm{ff}$.

53 Vgl. enwa Rolshausen, Macht und Herrschaft, 1997, S. 102 ff.; treffend in Arlehnung an Max Weber S. 149: "Da der 'Herre, dem dic Bürokracie dient, ein >Dilettant، ist, und der Stab auf arbeitsteiliger Grundlage ein Gruppenbewußtsein ausbilder, wird das Abhängigkeitsverhältnis schließlich umgekehrt: Die Bürokratie herrscht $\%$.

\$4 March/Olson, Rediscovering lnstitutions. The Organizational Basis of Politics, 1989; Becker, Olfentliche Verwaltung. Lehrbuch fur Wissenschaft und Praxis, 1989; Ortmann, Formen der Produktion. Organisation als Rekursivität, 1995; Ortmann/Sydow/lürk (Hrsg.), Theorien der Organisation, 1997.

ss Generell Luhmann, Sozialc Systeme, 1984; spezieller das Kapirel „Grenzen der Steuerungu in: Die Wirtschaft der Gesellschaft, 1988, S. $324 \mathrm{ff}$.

s6 Vgl. die Skizze des systemorienticrten Ansatzes in der Betriebswirtschaftslehre bei Pfriem, Unternehmenspolitik in sozialokologischen Perspektiven, 1995 , S. 135 ff.; zuletzt Schneidewind, Die Unternehmung als strukturpolitischer Akteur, r 998 .

s7 Ents. 9.6.1995, PersR 1996, 249 ff., 254. 

Maschinenmodells»

Der 6. Senat des BVerwG zeigt sich indes, wie bereits zuvor der Zweite Senat des BVerfG, von derartigen Einwänden unbeeinflußt. Dies mag neben der durchgängigen Empirieferne der Argumentation seinen Grund darin haben, daß die Gerichte davon ausgehen, daß parallel zur ununterbrochenen Legitimationskette vom Volk über die Regierung bis hin zum zuständigen Amtswalter ein gleichlaufender fachlicher Weisungsstrang besteht, der garantiert, daß bis in die Details jeder cinzelnen Behördenentscheidung hinein sich der zum Staatswillen geronnene Volkswille durchsetzen kann. Auf diese Weise würde die Verwaltung umfassend von einer gesellschaftlichen Zentrale aus gesteuert und könnten der Regierung alle noch so dezentralen Entscheidungen wie eigene zugerechnet werden, so daß sie für sie auch rechenschaftspflichtig und verantwortlich wäre.

Bereits in seiner ersten Grundsatzentscheidung zum Personalvertretungsrecht vom 27.4. $1959^{58}$ hat das BVerfG aus der parlamentarischen Verantwortlichkeit der Regierung - und damit aus dem Rechtsstaatsgebot, nicht etwa aus dem Demokratieprinzip - abgeleitet, daß bestimmte Regierungsaufgaben wegen ihrer politischen Tragweite nicht gcnerell der Regierungsverantwortung entzogen und auf von Regierung und Parlament unabhängige Stellen übertragen werden dürfen. Dieser Grundsatz ist anläßlich der Novelle des BPersVG vom 1 5.3.1974 ${ }^{59}$ in der rahmenrechtlichen Bestimmung des $\ \mathrm{ro4}_{4}$ S. 3 BPersVG kodifiziert und dahingehend kanonisiert worden, daß "Entscheidungen, die wegen ihrer Auswirkungen auf das Gemeinwesen wesentlicher Bestandtcil der Regierungsverantwortung sind ... nicht Stellen entzogen werden (dürfen), die der Volksvertretung verantwortlich sind “. Gestützt auf diese Formel wurde in $\$ 10_{4}$ S. 3 BPersVG cin echtes Mitbestimmungsrecht der Personalvertretung mit Letztentscheidungsbefugnis der Einigungsstelle insbesondere in personellen Angelegenheiten der Beamten und in organisatorischen Angelegenheiten ausdrücklich rahmenrechtlich ausgeschlossen. Mit der Wendung zu der von Bökkenförde entfalteten demokratietheoretischen Legitimationstheorie der Verwaltungssteuerung wurde nicht nur der rechtsstaatliche Begründungskontext ausgewechselt, sondern der Anwendungsbercich dieses "Regierungsvorbehalts « auf jegliches "amtliche Handeln mit Entscheidungscharakter " universalisiert. Folglich darf sich nach der Entscheidung des Zweiten Senats des BVerfG eine effektive Mitbestimmung nur noch "auf innerdienstliche Maßnahmen erstrecken und nur so weit gehen, als die spezifischen in dem Beschäftigungsverhältnis angelegten Interessen der Angehörigen der Dienststelle sie rechtfertigen $4 .{ }^{60}$ Aber auch insoweit gilt: "Auch im internen Dienstbetrieb ist kein Raum für eine >Autonomier des öffentlichen Dienstes, sei diese auch noch so eingeschränkt ${ }^{61}{ }^{61}$

s8 BVerfGE 9, 268 ff., 287.

\$9 Zur Karriere dieser Formel vgl. die Darstellung bei Aitvater/Bacher/Horter/Peiseler/Sabottig/Schneider/ Vohs, BPersVG, Kommentar, 4. Auff., 1996, \$104 Rn. i 2 ff.

60 BVerfGE 93, $37 \mathrm{ff} ., 70$.

6r BVerfGE 93, 37 ff., 70. In den aufgrund dieser Entscheidung inzwischen erfolgten Novellierungen einiger Landespersonalvertetungsgesetze (Niedersachsen, Sachsen-Anbalt, Sachsen) wird das Letztentschcidungsrecht der Einigungsstelic z. T. noch erheblich weitergehend cingeschränkr. Die extremste Konsequenz aus dieser Rechtsfortbildung, vonach die Souveränität der Regierung nicht nur in Grundsatzfragen des Gemeinwohls, sondern generell bei ihrer Ausubung von Staatsgewalt nicht durch * Dritte beschrankt werden darf, hat der Freistaat Sachsen bei seiner Änderung des Personalvertretungstechts gezogen. Danach sind gar sämcliche, zwischen Personalrat und Dienststelle abgeschlossenen Dienstvereinbarungen unter den Vorbehalt ihrer jederzeitigen, einseitigen Aufhebbarkeit durch die Dienststellenleitung gestells worden. Getreu der absolutistischen (= legibus solutus) Maxime, daß der souveräne Wille nur dann wirklich frei ist, wenn er jederzeit neu entscheiden kann, duldet er nicht einmal mehr die Beschränkung 
Bei genauerer Betrachtung erweist sich jedoch, daß der personellen Legitimation das Primat in dieser demokratischen Rechtfertigungslehre keineswegs um ihrer selbst willen zukommt. Vielmehr erhält dieses Legitimationsmuster diese zentrale Rolle nur, um der inhaltlichen Weisungsbefugnis der übergeordneten Stellen Durchschlagskraft zu verschaffen. Dies wird deutlich an den Ausführungen des Zweiten Senats des BVerfG zur nicht hinreichenden demokratischen Legitimation der Einigungsstelle, die entscheidend "aus der ihr durch $\$ 5_{3}$ Abs. 3 S. 3 Mitbestimmungsgesetz SchleswigHolstein gewährten Weisungsfreiheit $\alpha^{62}$ und der Weisungsfreiheit der von der Dienststelle bestellten Beisitzer der Einigungsstelle bei der Wahl des Vorsitzenden ${ }^{63}$ folgt. Das gleiche Muster läßt sich an den Vorlagebeschlüssen des BVerwG v. 17. 1 2. $1997^{64}$ ablesen. Das Gericht entnimmt den beiden Wasserverbandsgesetzen mit seinen "global-handlungssteuernden Regelungen" und der "detailliert und umfassend geregelte(n) staatlichen Aufsicht, die neben der Rechtsaufsicht auch Ansätze einer Fachaufsicht einschließt«, zwar eine "gesteigerte Gesetzesbindung und die demokratisch verantwortete Aufsicht über die Einhaltung diescr Bindung ". ${ }^{6 s}$ Aber auch dieses durchaus hohe sachlich-inhaltliche Legitimationsniveau genügt nicht, weil es an »konkreten oder gar exakten Zielvorgaben für das Handeln der Genossenschaftsorgane ${ }^{66}$ fehlt. Im Kern ist es dieser Mangel an durchgängiger Abhängigkeit von fachaufsichtlichen Weisungen, der das Demokratiedefizit begründet. ${ }^{67}$ Die personelldemokratische Legitimation und ihre lückenlose Kette stellt folglich nur die personalrechtliche Schiene dar, auf der die inhaltlichen Weisungsstränge laufen. Die Weisungsgebundenheit aller Amtswalter ist in dieser Demokratietheorie der Weisheit letzter Schluß. Dafür genügt es aber nicht allein, daß die Verwaltung entsprechend dem Modell klassisch-bürokratischer Herrschaft hierarchisch aufgebaut ist. Vielmehr muß das Funktionieren der "sachlich-inhaltlichen" Befehls-Gehorsams-Pyramide auch durch eine umfassende Etablierung detailgenauer fachaufsichtlicher Befugnisse in den Errichtungsgesetzen der einzelnen Verwaltungseinheiten und durch eine entsprechende Ausgestaltung des Personalrechts, insbesondere des Direktionsrechts der

durch kollektivrecheliche Selbstbindung. Im Ergebnis lauft diese Art der Verwaltungsmodernisierung auf die Aufhebung der Personalratsmitbestimmung hinaus, die wesentlich durch den Abschluß von Dienstvereinbarungen ausgeübt wird. Dieser mitbestimmungsrechtliche Kahlschlag licgt insoweit im Bannkreis der Logik des Beschlusses des Zweiten Senats des BVerfG, als danach »das Letzrentscheidungsrech ... vom Grundsatz her nicht uneingeschränkt gewàhrt, sondern im Einzelfall an die Voraussetzung geknuipft (wird), daß der Beschluß der Einigungsstelle wegen seiner Auswirkungen auf das Gemeinwesen die Regierungsverantwortung wesentlich beruhrt * (E 93, 66 ff., 79). Das entscheidende Stichwort lauter: Im Einzelfall $\alpha$. Wenn die Auswirkungen ciner Entscheidung stets im Enzelfall neu zur Debatte stch.en, dann mag die Dienststelienleitung eine generelle Vercinbarung in Fall a heute so und morgen im Fall $b$ cben anders beurtcilen. Fallt die Beurteilung im Fall $b$ anders aus, dann muß die Regelung kraft Regierungsverantwortung auch aufgehoben werden können. Das Dictum von Carl Schnitt: "Souverän ist, wer uber den Ausnahmezustand entscheider $\propto$, lautet in zeitgemäßer Reformulierung: Souverän ist, wer über den Einzelfall entscheidec.

62 BVerfGE $93,37 \mathrm{ff} ., 77$

63 "Die beiden von der Dienststelle bestellten Beisitzer sind ihrerseits zwar hinreichend personell demokratisch legitimiert, weil sie ihr Amt von einem seinerseits demokratisch legitimicrten Amtsträger ableiten. Da sic aber weder parlamentariseh verantwortich handeln noch dem Weisungsrecht eines parlamentarisch verantworlichen Amtsträgers unterliegen, kann ihre Mitwirkung bei der Bestellung des Vorsitzenden diesem ebenfalls keine uneingeschränkte personelle demokratische Legitimation vermitteln $\propto$ (ebd.).

$646 \mathrm{C}_{1}$ und 2.97 .

65 EmschGG, 6 C 2.97, Umdr. S. 29.

66 EmschGG, 6 C 2.97 , Umdr. S. 29.

67 Folglich kommt das Gericht bei sciner Skizzierung der Organisationsalternativen, die dem Gesetzgeber bei der Bildung der Wasserverbande zur Verfügung standen und bei der u. U. gebotenen Neufassung der Gesetze zur Verfügung stehen werden, zu dem Ergebnis, daß entweder "die Aufgabe der nicht individuell Icgitimierten Amtswalter ... auf eine im wesentliche beratende Funktion reduziert w werden müsse oder aber der Staat die "Aufgabe durch einen Teil der Landesverwaltung e erfüllen könne (EmschGG, $6 \mathrm{C}_{2.97}$, Undr. S. 30). 
Vorgesetzten und die Sanktionierung von Abweichungen, sichergestellt werden. Das verfassungsgerichtliche Demokratiemodell effordert demnach weit mehr als nur eine spezifische Organisationsstruktur der Verwaltung: Es verlangt außerdem einen spezifischen Typus konditional-programmierender Verwaltungsgesetze und ein auf die Erfüllung der Gehorsamspflicht spezialisiertes Personalrecht.

Dieses Modell ist in seiner Weltfremdheit und in seiner Schlichtheit gefährlich: Wenn schon das "absolutistische Maschinenmodell ${ }^{68}$ einer Programmierung der Verwaltung auf dem relativ abstrakten Niveau gesetzlicher Direktiven, wie sie etwa das BVerwG den beiden Wasserverbandsgesetzen entnimmt, als Paradigma rechtlicher (Verwaltungs-)Steuerung theoretisch wie praktisch überholt ist, dann gehört das Konzept einer inhaltlichen Detailprogrammierung aller relevanten Verwaltungsentscheidungen per fachlicher Einzelweisung erst recht in das Reich juristischer Fabelwesen. Wie Verwaltungseinheiten strukturiert sind, in welchen Programmformen sie gesteuert werden und welche Instrumente hierbei typischerweise eingesetzt werden und jeweils besonders effektiv sind, hängt entscheidend von der jeweils zu erfüllenden Verwaltungsaufgabe ab. ${ }^{69}$ Wenn es richtig ist, daß die Verwaltung der Bundesrepublik Deutschland »den Eindruck eines nahezu undurchschaubaren Geflechts von Verwaltungseinheiten, einer kaum zu entwirrenden Vielfalt an Gliedern, Organen, Ämtern und Institutionen erweckt ${ }^{70}$ und $»$ sich als hochgradig partikulares, zersplittertes, arbeitsteiliges Gefüge unterschiedlichster Teileinheiten, die große Unterschiede im Aufgabenbereich, in der Binnenorganisation, in Kontrollierbarkeit und Rechtsstatus aufweisen " ${ }^{71}$, dann ist es methodisch geboten, "Rechtsformen oder Organisationsprinzipien nicht absolut zu setzen, als ständig und überall passendes Allheilmittel zu behandeln und verfassungsrechtlich als allein sselig machend $z u$ überhöhen. Rechtsformen wie Organisationsprinzipien sind kein Zweck an sich, sie sind Mittel und Instrumente zur Erreichung bestimmter Zwecke, der Gewährleistung demokratisch verfaßter Staatlichkeit. Sind Rechsformen und Organisationsprinzipien Instrumente, so sind sie - und das ist der zentrale Punkt - problemspezifisch und bereichsspezifisch möglicherweise in unterschiedlichem Maße geeignet und daher auch verfassungsrechtlich zulässig oder unzulässig. ${ }^{73}$

Entsprechend dieser Maxime gehen denn auch nahezu alle neueren monographischen Untersuchungen ${ }^{73}$ zum Verhältnis von Demokratieprinzip und Verwaltungsorganisation dahin, die Gründe für die Abweichungen vom absolutistisch-hierarchischen Modell der Verwaltung in der breiten Palette der sog. ministerialfreien Räume, der mittelbaren Staatsverwaltung, der funktionalen Selbstverwaltung, der öffentlichen Kultureinrichtungen oder der öffentlichen sowie der gemischt öffentlich-privaten Unternehmen zu analysieren und normative Rechtfertigungen für die Abweichungen vom Typus der Ministerialverwaltung zu entfalten, die ihrerseits unter massivem Veränderungsdruck steht und sich intern in Richtung auf verstärkte "Flexibilität", Dezentralisierung, Eigenverantwortung, "Diskursivität«, "Mitarbeiterbeteiligung «

68 Drcier (Fn. Sr), S. 212.

69 Eingehend hierzu Schuppert (Fn. 18), S. 587 ff., 601; Konig/Dose, Klassificierungsansatze staatlicher Handlungsformen. Eine steuerungstheoretische Abhandlung, 1989.

70 Dreier (Fn. S1), S. 219.

71 Dreier (Fn. SI), S. 219 F.

72 Schuppert (Fr. 18), S. 606

$73 \mathrm{Vgl}$. Ehlers, Verwaltung in Privatrechtsform, 1984; Oebbecke, Weisungs- und untcrrichtungsfreie Raume in der Verwaltung, 1986; Dreier, Hierarchische Verwaltung im demokratischen Staat, 1991; Emde, Dic demokratische I.egitimation der funktionalen Selbstverwaltung, 1991; Wacchter, Geminderte demokratiche Legitimation staarlicher Institutionen im parlamentarischen Regierungssystem, 1994; Plander, Personalvertretungen als Grundrechtshilfe im demokratıschen und soziaien Rechtsstaat, I995; auf dem Boden der dogmatisierten Demokratietheorie dagegen Jaestedt, Demokratieprinzip und Kondominialverwaltung, 1993, der Ausnahmen vom Typus der Ministerialverwaltung nur soweit für zulässig erachtet, wie sie im GG selbst vorgesehen sind. 
und "Kontraktmanagement « reorganisiert und zu den Adressaten hin öffnet. ${ }^{74}$ Grob typisiert lassen sich für die partielle Autonomisierung von Verwaltungseinheiten drei Rechtfertigungsmuster"s identifizieren: Rechtsstaatliche Gründe wie spezielle Kontroll- und Aufsichtsfunktionen ${ }^{76}$ ( $z$. B. Rechnungshöfe, Bundesschuldenverwaltung, Bundesbank, Finanzminister, Bundeskartellamt), kommunikativ-kulturelle Grundrechte von Akteuren wie Adressaten, die eine staatsferne Form dieser Insitutionen erfordern ${ }^{77}$ (z. B. Rundfunk- und Fernsehanstalten, Staatstheater, Wissenschaft und Forschung, Filmbewertungsstelle Wiesbaden, Bundesprüfstelle für jugendgefährdende Schriften, Filmförderungsanstalt) sowic persönliche Freiheits- und soziale Entfaltungsgrundrechte von Betroffenen, Mitgliedern sowie Beschäftigten entsprechender Verwaltungseinrichtungen als Grundlage der ihnen eingeräumten Selbstverwaltung oder Partizipation ${ }^{78}$ (z. B. die Betroffenen- und Arbeitnehmermitbestimmung im Gesamtbereich der funktionalen Selbstverwaltung wie der Wasserverbände, Kammern und Innungen und der öffentlich-rechtlich organisierten Wirtschaftsunternehmen wie der Sparkassen und Versicherungen sowie den großen Sektor der Sozialversicherungsträger). Es sind damit nichts weniger als die grundlegenden Prinzipien der Verfassung selbst, das Rechtsstaats-, Demokratie- und Sozialstaatsprinzip, die diese Abweichungen vom "absolutistischen « Normalmodell der Verwaltungsorganisation tragen.

Bereits Max Weber hat den Versuch, aus dem demokratischen Volkswillen Organisationsprinzipien der Verwaltung abzuleiten, treffend wie folgt charakterisiert: "Begriffe wie , Wille des Volkes<, wahrer Wille des Volkes usw. ... sind Fiktionen. Es ist gerade so, als ob man von einem Willen der Stiefelkonsumenten reden wollte, der für die Art, wie der Schuster seine Technik einrichten sollte, maßgebend sein müsse! «79 Dieser »Ableitungspurismus « sieht in geradezu fundamentalistischer Selbstgewißheit von allen Besonderheiten der jeweils anstehenden Sachmaterien ab. Dies ist umso weniger nachvollziehbar, als durchaus einschlägige, vergleichende empirische wie normative Studien (und einschlägige Verfassungsgerichtsentscheidungen!) zu den Effekten der Arbeitnehmermitbestimmung ${ }^{80}$ oder im Bereich der Wasserwirtschaft zur Eignung der unterschiedlichen Träger (staatliche Verwaltung, die bestehenden Wasserverbände oder private Unternehmen) für die Erfüllung der wasserwirtschaftlichen Aufgaben bestehen. ${ }^{81}$ Hinsichtlich dieser letzteren Frage erweist sich, daß

74 Vgl. hierzu Blanke, Verwaltungsmodernisierung, 1998 mit zahlr. Nachw.

75 Andere unterscheiden anders. Wagner z.B. kommt auf ${ }_{13}$ Gründe für die Verselbständigung von Verwaltungsorganisationen, vgl. ders., Typen der verselbstandigten Erfüllung öffentlicher Aufgaben, in: ders. (Hrsg.), Verselbständigung von Verwaltungstrgagern, 1976, S. 245 ff., 262; Dreier (Fn. 51), S. 270 ff. typisiert schwerpunktmäßig zwei Motive: Verfassungsrechtliche Direktiven und funktionale Aspekte; ähnlich wie hier z. B. Krebs: Entpolitisienng (z. B. Bundesbank), Staatsferne (Rundfunkanstalten), grundrechtliche Freiräume (Hochschulen) und die Idee der Selbstverwaltung, vgl. ders., Verwaltungsorganisation, in: HdbStR IIl, $\oint_{59}$ Rn. $69 \mathrm{ff}$.

76 Diesc entfaltet insbesondere die Arbeit von Waechter (Fn. 73); Dreier spricht insoweit bei Verwaltungsentscheidungen mit quasi-judizieller Funktion von der Distanzierungsfunkcion.

77 Hierzu näher Oebbecke (Fn. 73), S. $192 \mathrm{ff}$.

$78 \mathrm{Vgl}$. insbesonderc Emde (Fn. 73), S. $354 \mathrm{ff}$., $426 \mathrm{ff}$., 452 ff.; Ehlers (Fn. 73), S. 3 I s; für die Personalvertretung ausführlich Plander (Fn. 73).

79 Zit. nach Neumann, Demokratietheorien (Fn. 20), S. 53.

8o Vgl. Schneider, Die Mitbestimmung im öffentlichen Dienst - ein geschichtlicher Rückblick, RiA I986, I 72 ff.; ders., Wirtschaftliche Mitbesummung in öffentlichen Unternehmen, DÖV 1972, 589 ff.; Plander, Personalvertretungen als Grundrechishilfe im demokratischen und sozialon Rechtsstaat, 1995; Steincr, Der besondere Stellenwert der Mitbestimmung im offentlichen Dienst vor dem Hintergrund der Entwicklungsgeschichte gesetzlich normierter Mitbestimmungsregclungen, PersV I 986 , 143 ff.; zur Diskussion zu Beginn der yocr Jahre vgl. Bicdenkopf/Sacker, Grenzen der Mitbestimmung in kommunalen Versorgungsunternehmen, ZfA 1991, 2 I Iff; aus der verfassungsgerichtlichen Rechtsprechung vgl. dic Mitbestimmungsentscheidung BVerfGE $50,290 \mathrm{ff} ., 350 \mathrm{f}$.

81 V $\mathrm{gl}$. z. B. Endres/Holm-Müller, Die deutschen Wasserverbände als Vorbild für Umweltgenossenschaften? Eine ökonomische Betrachtung, in: Endres/Marburger, Umweltschutz durch gesellschafeliche Selhst- 
keine dieser Organisationsformen für sich genommen einen genuinen Platzvorteil

besitzt, sondern alle nur unter spezifischen Zusatzbedingungen optimal im Sinne effizienter, kostensparender, sozialer und umweltschonender Leistungserbringung funktionieren. Insgesamt zeichnet sich aber ab, daß die erforderlichen Steuerungen besonders effektiv, flexibel und effizient und damit wohl am besten bei einer Mischung aus öffentlich-privater Leistungserstellung greifen, wobei die Systeme für beständige Nachkorrekturen offen sein müssen. ${ }^{82}$

Eine nach dem Modell der Ministerialverwaltung durchgängig auf Hierarchie, personelle Legitimationsketten und fachliche Weisungsstränge umgestellte Organisation aller bundesdeutschen Verwaltungseinheiten wäre aber auch gefährlich - und zwar gleichermaßen unter Legitimationsaspekten wie unter Effizienzgesichtspunkten. Wenn in Gesprächen unter Fachleuten die demokratische Legitimationstheorie der Judikatur in der Weise auf den Begriff gebracht wird, daß demnach nicht der von den Mitschülern gewählte, sondern nur der vom Schulleiter ernannte Schulsprecher demokratisch legitimiert sei, und diese Pointe als Konsequenz und nicht als Karikatur dieser Auffassung gewertet wird, dann wird deutlich, wie der vom Volk ausgegangene demokratische Wille zum Volk zurückkehrt: als obrigkeitlicher Befehl, Ernennung und Weisung. Konkrete Legitimationsverluste "vor Ort« sind die Folge.

Unter Elfizienzgesichtspunkten ist zu befürchten, das das hierarchische Verwaltungsmodell bereits nach kürzester Zeit daran scheitern würde, daß eine solche "Trivialmaschine « der Komplexität ihrer Umwelt mangels entsprechend hoher interner Komplexität nicht gewachsen wäre: „Die verselbständigten Verwaltungseinheiten erweisen zunächst einmal in aller wünschenswerten Deutlichkeit und Eindringlichkeit, daß die der öffentlichen Verwaltung obliegende Aufgabenvielfalt im modernen demokratischen und sozialen Verfassungsstaat sich nicht in den Erfüllungs- bzw. Funktionsmodus strikt hierarchisch-bürokratischer Organisation mit weisungsabhängiger Ausführung vorgegebener Normenprogramme pressen läßt. In einer Reihe von je spezifisch zu legitimierenden Bereichen bedarf es zur sachadäquaten Aufgabenerfüllung ganz im Gegenteil der Herausnahme aus dem hierarchisch-bürokratischen Kontroll und Entscheidungssystem ${ }^{83}$ Ruinöses Anschauungsmaterial hinsichtlich der Folgen einer hierarchisch entdifferenzierten »Kommandoverwaltung « ist in Deutschland genug zu besichtigen. Es sollte nicht deshalb vermehrt werden, um einer verkürzten, privatistischen Vorstellung - und sei es auch namhafter Staatsrechtslehrer und hochrangiger Gerichte - über die verwaltungs-

sreuerung, 1993, S. 16 Iff.; Batley, Public-Private Relationships and Performance in Service Provision, Urban Studies, 1996, Nr. 4/5, S. $723 \mathrm{ff}$.; Nickson, The public-private mix in urban water supply, International Review of Administrative Sciences, 1997, H.3, S. 16s ff.; Scheeic, Privatisierung der britischen Wasserwirtschaft, in: Zeitschrift für offentlichc und gemeinwirtschaftliche Unternehmen, Bd. $14, \mathrm{H} .4$, S. 346 ff.; ders., Kommunale Infrastrukturen. Die Sicherung offentlicher Interessen in cinem deregulierten Umfeld, Gutachten fur die Hans-Bockler-Stiftung, 1998 (unveroff.); Spelthahn, Privatisicrung natürlicher Monopole. Theorie und internationale Praxis am Bcispiel Wasser und Abwasser, Wiesbaden 1993; Stihl, Liberalisienung der Märkce. Wettbewerb auch in der Wasserwirtschaft? Der Städtetag tgg8, S. $331 \mathrm{ff}$.

82 Zuletzr eingehend Scheele (Fn. 81 ) 5998 , S. ss ff.; Endres/Holm-Muller (Fn. 81), S. i 81 betonen dagegen starker die Autonomieerfordernisse, wenn sie resumieren: $\rtimes$ Besonders vorteillzaft waren Umweltgenossenschaften dann, wenn sie ihre Aktivitaten nicht parallel zu den staatlichen Organen entfalten, sondern diese zumindest in Teilbercichern ersetzen*. Ein interessantes Beispiel für die Offenheir für Nachkorrekturen liefere die Privatisierung der Wasserwirtschaft in Großbritannien. Dadurch sind durchaus erhebliche Effektivitatsverbesserungen bewirkt worden, allerdings verbunden mit größeren sozialen Disparicaten. Diesen normativen Defiziten begegnet dic Labour-Regierung scit ihrem Amtsantritt mit verstarkten ReRegulierungsmaßnahmen durch die Aufsichtsbehörden. Mittelfristig scheint aber selbst das »natürliche Monopolø Wasser kein Hindernis mehr fur die Einfuhrung von effektiven Wetthewerbsstrukturen zu bedeuten, vgl. Stihl (Fn. 81), S. 331.

83 Dreier (Fn. S1), S. 297. 
organisatorischen Konsequenzen des Demokratieprinzips in einer modernen Gesellschaft traurige Gestalt zu verleihen.

\section{Zusammenfassung}

Die dominierende verfassungs- und verwaltungsgerichtliche Judikatur leitet die Selbstverwaltungs- und Mitbestimmungsschranken im Bereich staatlichen Handelns zwar aus dem Demokratieprinzip und damit aus dem Gedanken der Volkssouveränität ab. Im Effekt erweitert sie dadurch jedoch nicht ctwa den Einfluß des im Parlament repräsentierten Souveräns auf die organisatorische Ausgestaltung und personelle Ausstattung der Verwaltung, sondern entzieht ihm die politisch-demokratische Prärogative durch die Festschreibung eines autoritär-obrigkeitlichen Verwaltungsmodells mit strikter fachlicher Weisungsgebundenheit. Dadurch werden die Amtsträger, ganz im Gegensatz zu den verbalen Bekundungen dieser Lehre, nicht etwa an die Entscheidungshoheit des Parlaments gebunden. Vielmehr wird ihre Verselbständigung gegenüber dem parlamentarischen Gestaltungswillen forciert. In den praktischen Auswirkungen bedeutet dies nicht nur eine Ausgrenzung der mitbestimmungsfreundlicheren sozialdemokratischen Demokratiekonzeption, die in den vielfältigen Bemühungen sowohl des Bundesgesetzgebers bei der Neufassung des BPersVG v. t 5. 3. $1974^{8_{4}}$ als auch der Landesgesetzgeber (z. T. mit Unterstützung der GRÜNEN) zur Ausweitung der Personalratsmitbestimmung zum Ausdruck gekommen ist, aus dem vielbeschworenen Verfassungskonsens. ${ }^{85}$ Deurlich wird zugleich das Versagen der herrschenden bundesdeutschen Staatsrechrslehre, ein der liberalen, sozialen und demokratischen Tradition des GG entsprechendes Demokratieverständnis zu entfalten, das die Selbstverwaltungsidee des 19 . Jahrhunderts fortentwickel ${ }^{86}$ und Anschluß an die westeuropäischen wie insbesondere angloamerikanischen Demokratievorstellungen sucht. ${ }^{87}$ Damit widerspricht dieses Demokratiemodell nicht nur modernem westlichen Verfassungsverständnis, sondern hemmt auch die Erfüllung des Verfassungsauftrags aus Art. 23 Abs. I S. 1 GG zur »Verwirklichung eines vereinten Europas«.

Das Demokratieprinzip fordert in der Logik der Entscheidung des Zweiten Senats des BVerfG vom 24. 5. $1995^{88}$ diesen und nur diesen Verwaltungsaufbau: Daran kann nicht einmal ein Parlamentsgesetz etwas ändern. Damit ist - im Namen des Demokratieprinzips und angeblich um der Souveränitär des Parlaments willen - diesem die Befugnis entzogen, über die Formen der Verwaltungsorganisation selbst entscheiden zu können. So wird das Demokratieprinzip zur Fessel der Demokratie. Diese Lesart des Demokratieprinzips entmündigr den demokratischen Gesetzgeber und verwandelt die Staarsbürger wie die Beschäftigten des öffentlichen Dienstes in Untertan der obrigkeitsstaatlich gegliederten Verwaltung. In konsequenter Anwendung der Grundsätze der Entscheidung des BVerfG v. 24. 5. 1995 binden die Vorlagebeschlüsse des $B V e r w G^{\delta 9}$ darüberhinaus die Verwaltung an ein - wenn nicht insgesamt überholtes, so doch eng begrenztes - Organisationsmodell von Verwaltung. Dieses hat mit den Strukturen, die mit allseitiger politischer Unterstützung landauf, landab im Zuge

84 BGBL. I S. 693

8s Vgl. hierzu Blanke, PersR 1996,222 f.

86 Vgi. hierzu ausfuhrlich Emde (Fn. 73), S. 383 ff., 398 ff., 4 roff.

87 Dazu vgl. Bryde (Fn. r 8)

88 BVerfGE $93,37 \mathrm{ff}$.

89 Beschlüsse v. 17. 12.1997, Az. $6 \mathrm{C}$ I und 2.97 . 
der Verwaltungsmodernisierung eingeführt werden, nichts gemein. Ähnlich weit entfernt von der Realität ist angesichts der laufenden Privatisierungswelle, die selbst vor den Institurionen des Strafvollzugs ${ }^{9 \circ}$ nicht halt zu machen gedenkt, das Dictum in den Vorlagebeschlüssen des BVerwG, daß der Gesamtbereich, in dem der Staat wichtige Schutzaufgaben zu übernehmen habe und in dem Staatsgewalt ausgeübt werde, »letztlich " privatisierungsfest sei.9"

Als Fazit bleibt die Erkentnis: Mit normativen Mitteln allein lassen sich Organisationsfragen nicht sinnvoll entscheiden. Deshalb führt jeder Versuch, aus dem Demokratieprinzip eine verbindliche Form der Verwaltungsorganisation abzuleiten und daraus Grenzen für die Zulässigkeit der Mitbestimmung der Beschäftigtenvertretung sowie der Einbeziehung von Privaten in die Erledigung öffentlicher Aufgaben zu entwickeln, gleichermaßen zur Dogmatisierung wie zur Verfehlung der Realität. Dadurch werden dem parlamentarischen Gesetzgeber Optionen genommen, die ihm im Interesse der Demokratie erhalten bleiben müssen. Denn die Festlegung einer einzig zulässigen Form der Erledigung öffentlicher Aufgaben führt zu einer drastischen Verengung des politischen Gestaltungsspielraums auf die Alternative: Entweder hierarchisch-weisungsgebundene Verwaltung oder Privatisierung. Daß angesichts dieser $Z$ wangserstarrung der Staatsorganisation allenthalben die Staatsflucht angetreten wird, kann nicht länger verwundern. Man sollte nur wissen: Sie ist hausgemacht und deshalb auch revidierbar. Zumindest dies folgt aus dem Demokratieprinzip.

Wolfgang Hoffmann-Riem/Eberhard Schmidt-Aßmann (Hrsg.)

\section{Effizienz als Herausforderung an das Verwaltungsrecht}

Dieser Band zeigt an ausgewählten Bereichen, wie eine schon aus finanziellen Erwägungen geforderte Effizienz als Anstoß zu überfälligen Reformen in Verwaltungsorganisation und Verwaltungsrecht dienen kann.

1998, 276 S., geb., 72,- DM, 526,-öS, 65,50 sFr, ISBN 3-7890-5187-X

(Schriften zur Reform des Verwaltungsrechts, Bd. 5)

$90 \mathrm{Vg}$ ! hierzu jungst den Initiativantrag der CDU am Hessischen Landtag sbetreffend Justizvollzugsan stalten in privater Trágerschaft«, Landtags-Drs.r $4 / 3822$ v. 22.4. 1998.

91 EmschGG, Az.: 6 C 2.97, Undr. S. 24. 